\title{
Influence of polar groups in binary polymer blends on positronium formation
}

\author{
P. Ramya, ${ }^{1}$ P. Guagliardo, ${ }^{2}$ T. Pasang, ${ }^{1}$ C. Ranganathaiah, ${ }^{1,2}$ S. Samarin, ${ }^{2}$ and J. F. Williams ${ }^{2, *}$ \\ ${ }^{1}$ Department of Studies in Physics, University of Mysore, Manasagangotri, Mysore-570006, India \\ ${ }^{2}$ Australian Research Council Centre of Excellence for Antimatter-Matter Studies, \\ Centre for Atomic, Molecular and Surface Physics, School of Physics, University of Western Australia, Crawley, WA 6009, Australia
}

(Received 2 March 2013; published 13 May 2013)

\begin{abstract}
The present work studied the role of the polar group unconjugated oxygen on the inhibition of positronium (Ps) formation in two binary blends made from a set of chosen constituent polymers with polar and weakly polar groups (nonpolar). The polymer blend samples of PVC-EVA and PVC-SAN were investigated by coincidence Doppler broadening and positron lifetime techniques. The strong polar acetate group in the EVA contributed to positron annihilation with electrons of unconjugated oxygen $\left(-\mathrm{C}^{+}=\mathrm{O}^{-}\right)$as revealed by the momentum distribution curves peaking around $17 P_{L}\left(10^{-3} m_{0} c\right)$. The ortho-Ps intensity indicated the unconjugated oxygen shows about a $28 \%$ Ps reduction even in the presence of a strong Ps inhibiting halogen $\left(\mathrm{Cl}^{-}\right)$. In contrast, this effect was not seen in the PVC-SAN blends since SAN contains a weakly polar (nonpolar) acrylonitrile group $(\mathrm{C} \equiv \mathrm{N})$. Our results indicate the chlorine of PVC in the blends is a major contributor to Ps inhibition through the formation of a $\left(\mathrm{Cl}^{-}-e^{+)}\right.$bound state but the unconjugated oxygen in EVA of the PVC-EVA blend also plays a similar, but lesser, role.

DOI: 10.1103/PhysRevE.87.052602

PACS number(s): 61.25.hk
\end{abstract}

\section{INTRODUCTION}

This paper concerns the application of positron annihilation techniques to identify the effects of polar groups with a dipole distribution of electric charge in polymer chains and the extent to which those effects may be modified in polymer blends [1-16].

We look specifically at the polar carbonyl group with oxygen double bonded to a carbon atom with a dipole moment $(-\mathrm{C}=\mathrm{O})$ to explore how positron trapping by polar groups, that are a negatively charged part of the dipole $\left(-\mathrm{C}^{+}=\mathrm{O}^{-}\right)$, inhibits Ps formation. The effects of a conjugated oxygen atom in a molecule of the type $\mathrm{C}=\mathrm{C}-\mathrm{C}=\mathrm{O}$ are compared with those of an oxygen atom in an isolated $-\mathrm{C}=\mathrm{O}$ unconjugated group and with those of halogen atoms.

Noting that a conjugated system contains overlapping (delocalized) $p$ orbitals across adjacent aligned $p$ orbitals which may bridge adjacent single bonds, two polymer blends were selected with different conjugation groups, in general with lower overall energy and different stability. We chose PVC-EVA (poly vinyl chloride-ethylene vinyl acetate) in which EVA contains a strong polar group of the type $-\mathrm{C}=\mathrm{O}$ (unconjugated) and PVC-SAN (poly vinyl chloride-styrene acrylonitrile) in which SAN is nearly nonpolar or very weakly polar in character due to the presence of the $\mathrm{C} \equiv \mathrm{N}$ group. According to the polar group concept the atoms with a difference in electronegativity less than 1.7 results in a covalent bond of a polar nature and when the difference is less than 0.5 a nonpolar covalent bond is formed. The carbonyl group $(-\mathrm{C}=\mathrm{O})$ in the acetate part of EVA shows a difference in the electronegativity between $\mathrm{C}$ (2.55) and $\mathrm{O}$ (3.44) of 0.89 indicating a strong polar group. For the SAN polymer, the cyanidyl $(-\mathrm{C} \equiv \mathrm{N})$ group is nonpolar or very weakly polar as the difference in the electronegativity between $\mathrm{C}(2.55)$ and $\mathrm{N}(3.04)$ is 0.49 . In both blends of this

*Corresponding author: jfw@physics.uwa.edu.au study, the PVC contains chlorine which is a strong inhibitor of Ps formation like other halogens. These polymer blends also have extensive applications in industrial and scientific fields requiring multifunctional and high performance materials without the limitations of single polymeric materials.

An understanding at the atomic level has direct significance. Coincidence Doppler broadening spectroscopy (CDBS) [17] and positron lifetime annihilation spectroscopy (PALS) are the preferred techniques for this study. Coincidence Doppler broadening spectroscopy is an established technique to measure and understand the chemical environment of materials at the site of positron annihilation with the measurement of momentum distributions of annihilation events with core/valence electrons. The well-established positron annihilation lifetime spectroscopy, particularly for polymers and polymer-based materials, concerns the free volume size and its distribution [18-26]. The high-momentum part of the Doppler-broadened annihilation spectra is used to distinguish different elements because the core electrons retain their atomic character even when atoms form a complex polymer. For example here, the relationship between the oxygen-containing groups and Ps annihilation can be identified and it also can identify elemental variations around a defect site. The next section considers the details of these experimental approaches.

\section{EXPERIMENT}

\section{A. Blend preparation}

Samples of PVC, SAN (25 wt. \% acrylonitrile), and EVA (40 wt. \% VA) with densities 1.34, 1.08, and $0.94 \mathrm{~g} / \mathrm{cc}$ and weight-average molecular weights 43000,165000 and $150000 \mathrm{~g} / \mathrm{mol}$, respectively, were procured from $\mathrm{M} / \mathrm{s}$ Sigma-Aldrich Chemicals Ltd. Bangalore. Blends of these samples were prepared by the conventional solvent-casting method. The weighed fractions of PVC and EVA were dissolved in the common solvent tetrahydrofuran at $60{ }^{\circ} \mathrm{C}$ in three different compositions (80:20, 50:50, 20:80) 
in a beaker. This beaker was then kept on a magnetic stirrer maintained at $60{ }^{\circ} \mathrm{C}$ and stirred continously with a magnetic bead in the beaker until the polymers were dissolved comlepletely and a homogenous solution obtained. The stirring was continued at room temperature until the solution equilibrated. Then this solution was cast onto a clean, flat glass plate and kept at room temperature to allow solvent evaporation for $24 \mathrm{~h}$ and film formation of about $1 \mathrm{~mm}$ thickness. The neat films were lifted from the glass plate and vacuum dried at $70{ }^{\circ} \mathrm{C}$ for about $10 \mathrm{~h}$ to remove any residual solvent. Complete solvent removal was confirmed by measuring constancy in the weight of the samples using a digital balance. A similar procedure was followed for making PVC-SAN blends of three compositions. All the samples were stored in a desiccator until used in the experiment.

\section{B. Coincidence Doppler broadening spectroscopy}

Conservation of energy and momentum of the annihilating positron-electron pair determines the scattering dynamics. The difference in the energy of the annihilation photons is equal to $c P_{L} / 2$, where $P_{L}$ is the longitudinal component of the positron-electron pair momentum along the direction of photon emission and $c$ is the speed of light, which is essentially equal to longitudinal component of electron momentum. Observing the coincidence between the two annihilation photons narrows the selection of photon momentum and leads to a significant reduction in the background [17]. The deduced electron momentum provides an accurate chemical environment sampled by the positron annihilation [10,27,28].

Line shape parameters describing the width of the annihilation line are typically employed to obtain qualitative information about electronic structure of solids. The $S$ parameter is defined as the area of the central part of the spectrum (low momentum) divided by the total area under the CDB spectrum. The $W$ parameter is taken in the high momentum regions far from the center. It is the ratio of the area under the wings in a fixed window divided by the total area. These regions are indicated in Fig. 1.

Another aspect of data treatment is to present the CDB spectral data as the ratio of the normalized counts in each

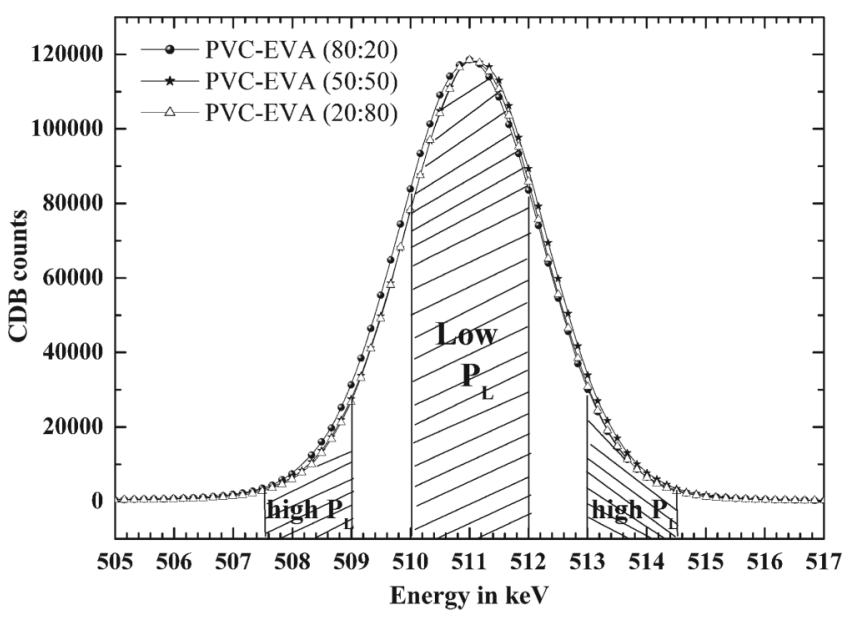

FIG. 1. Typical energy distribution curves of PVC-EVA polymer blend with varying blend composition. channel of the measured sample spectrum to the corresponding counts in reference sample spectrum [28]. The choice of the reference spectrum is important to determine the appropriate background calibration for the high momentum region of the sample spectrum.

The CDB measurements used a high purity germanium detector in coincidence with a $\mathrm{BaF}_{2}$ detector. The energy resolution of the $\mathrm{HpGe}$ detector was $1.5 \mathrm{keV}$ (full width at half maximum) at the $511 \mathrm{keV}$ photo peak of ${ }^{22} \mathrm{Na}$ and the channel width was $0.192 \mathrm{keV}$. Two identical samples of the blends were sandwiched between a positron source prepared by evaporating carrier-free ${ }^{22} \mathrm{NaCl}$ solution on Kapton foil of $7 \mu \mathrm{m}$ thickness. The source-sample sandwich was wrapped with $\mathrm{Al}$ foil and was placed at a distance of $20 \mathrm{~cm}$ away from each detector, $180^{\circ}$ apart. This distance minimized any increase in the background due to three-photon annihilation of the triplet state of o-Ps. The energies of the annihilating photon pairs in the range of energy $\left(m_{0} c^{2} \pm 2.4 \mathrm{keV}\right)$ were recorded by coincidence by the two detectors and an ORTEC multichannel analyzer. The CDB spectrum was recorded for about $15 \mathrm{~h}$ resulting in total counts of at least $15 \times 10^{6}$ counts under the annihilation photo peak at room temperature. The CDB spectra were analyzed using computer program SP-I to evaluate the $S$ and $W$ parameters. Furthermore, to construct momentum distribution of electrons with which positron and positronium annihilate, the $\mathrm{CDB}$ ratios were obtained by first normalizing the CDB counts of the blends and reference PVC counts and then taking the ratio.

\section{Positron annihilation lifetime measurements (PALS)}

The PALS technique and its instrumentation are well established [18-26]. Of relevance to polymers is the capture of an electron to form either para-positronium (p-Ps) if the positron and electron have aligned antiparallel spins or ortho-positronium (o-Ps) with parallel spins. In other than free space, o-Ps picks up an electron from the surrounding medium and its lifetime is shortened. This localized annihilation in the "free-volume holes" [18] makes it the microprobe of choice since its lifetime and intensity become a measure of the electron density and free-volume size in the study of microstructural behavior of polymers [18-26].

In the present work positron lifetime measurements were made using a standard fast-fast coincidence system with conically shaped $\mathrm{BaF}_{2}$ scintillators, coupled to photomultiplier tubes of type XP2020/Q with quartz window. The lifetime spectrometer had a time resolution of 220 ps. All measurements were made at room temperature. The sourcesample sandwich geometry was used for positron lifetime measurements in which the polymer sample was placed on both sides of the source in a sandwich configuration held together tightly by aluminum foil wrapping. The positron ${ }^{22} \mathrm{Na}$ source was about $2 \mathrm{~mm}$ diameter deposited at the center of a square Kapton foil of $12.7 \mu \mathrm{m}$ thickness. Details of the experimental procedure were given earlier [21,26].

Each spectrum contained at least $10^{6}$ counts and the instrumental time resolution and source correction terms were obtained from the lifetime spectrum of well-annealed aluminum, using the program RESOLUTION [29]. The lifetime spectra were analyzed using the PATFIT- 88 computer program 
TABLE I. PALS data of the PVC-EVA and PVC-SAN polymer blends.

\begin{tabular}{|c|c|c|c|c|c|c|c|}
\hline Sample & Composition & $\tau_{1} \pm 0.006 \mathrm{~ns}$ & $I_{1} \pm 1.8 \%$ & $\tau_{2} \pm 0.007 \mathrm{~ns}$ & $I_{2} \pm 1.9 \%$ & $\tau_{3} \pm 0.01 \mathrm{~ns}$ & $I_{3} \pm 0.3 \%$ \\
\hline PVC pure & 100 & 0.188 & 48.3 & 0.398 & 45.5 & 1.72 & 6.2 \\
\hline EVA pure & 100 & 0.174 & 42.4 & 0.425 & 36.8 & 2.46 & 20.8 \\
\hline SAN pure & 100 & 0.183 & 38.2 & 0.423 & 42.5 & 1.92 & 19.3 \\
\hline PVC-EVA & $20: 80$ & 0.169 & 36.6 & 0.383 & 44.4 & 2.29 & 19.0 \\
\hline \multirow[t]{2}{*}{ Blend } & $50: 50$ & 0.171 & 48.3 & 0.378 & 42.8 & 2.23 & 8.9 \\
\hline & $80: 20$ & 0.161 & 34.1 & 0.360 & 59.1 & 2.08 & 6.8 \\
\hline PVC-SAN & $20: 80$ & 0.180 & 35.1 & 0.414 & 46.2 & 1.92 & 18.7 \\
\hline \multirow[t]{2}{*}{ Blend } & $50: 50$ & 0.181 & 40.4 & 0.412 & 45.3 & 1.86 & 14.3 \\
\hline & $80: 20$ & 0.175 & 36.7 & 0.374 & 53.8 & 1.78 & 9.5 \\
\hline
\end{tabular}

[29] with proper source correction applied. For each sample at least three lifetime spectra were collected and analyzed and the average values of o-Ps lifetime and intensity are reported here. The reproducibility of the results was satisfactory and within the experimental error limits as in Table I. The finite term analysis of PALS decomposed the spectrum into three lifetime components $\tau_{1}, \tau_{2}$, and $\tau_{3}$ with intensities $I_{1}, I_{2}$, and $I_{3}$, respectively. The shortest lifetime $\tau_{1}$ with intensity $I_{1}$ corresponds to p-Ps and free positron annihilations. The second lifetime component $\tau_{2}$ with intensities $I_{2}$ is due to trapping of positrons at the defects and open volumes smaller than the free volume. The third component $\tau_{3}$ with intensity $I_{3}$ is ascribed to pick-off annihilation of the o-Ps from the free volume cavities present mainly in the amorphous regions of the polymer matrix.

\section{RESULTS AND DISCUSSION}

\section{A. CDBS results}

Figure 1 shows a typical annihilation energy spectra for polymer blends. The momentum distribution curves for PVCEVA and PVC-SAN blend samples (normalized counts) are

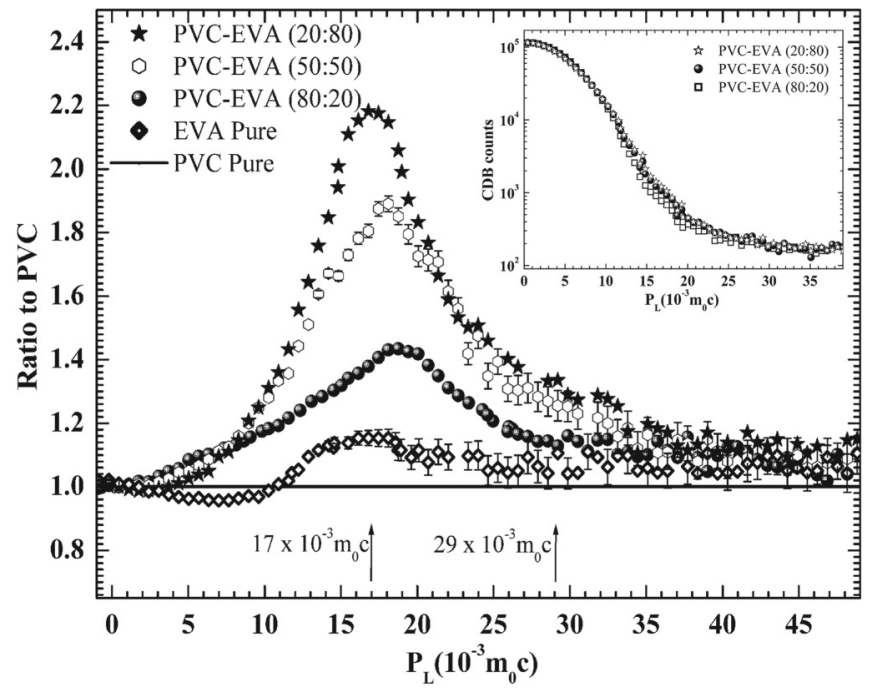

FIG. 2. Ratio curves for the CDB spectra of PVC-EVA blends and pure EVA with respect to pure PVC. In the inset CDB spectra of PVC-EVA blends with each spectrum normalized to the same total counts. shown in the inset of Figs. 2 and 3, respectively. To construct these curves the measured energy CDB spectra were analyzed as follows. First, the measured CDB spectrum in the blend and the reference PVC spectrum were normalized to the same peak counts. The energy axis ( $x$ axis) is expressed in momentum in units of $\left(10^{-3} m_{0} c\right)$. The inset of Fig. 2 shows these curves for PVC-EVA blends for different compositions. The curves are very similar except in the momentum region near 17 and 29 units. Similarly, the inset of Fig. 3 shows similar trends for PVC-SAN blends of different compositions except for the difference in the region of 16 and 29 units. A composition-dependent effect is seen (i) in particular as the chlorine content changes, which influences the inhibition of Ps formation and (ii) due to the nature of the electrons participating in the annihilation process. We constructed momentum distribution curves as the ratio of the normalized CDB counts in each channel of the measured spectrum of the blend to the corresponding channel $\mathrm{CDB}$ counts in a reference PVC spectrum. Then

$$
\text { Ratio }=N^{b}\left(P_{L}\right) / N^{r}\left(P_{L}\right),
$$

where $N$ represents the normalized CDB counts, $b$ denotes blend, $r$ denotes reference, and $P_{L}$ runs from 0 to $45 \times$ $10^{-3} m_{0} c$. For both blend systems, the PVC sample was

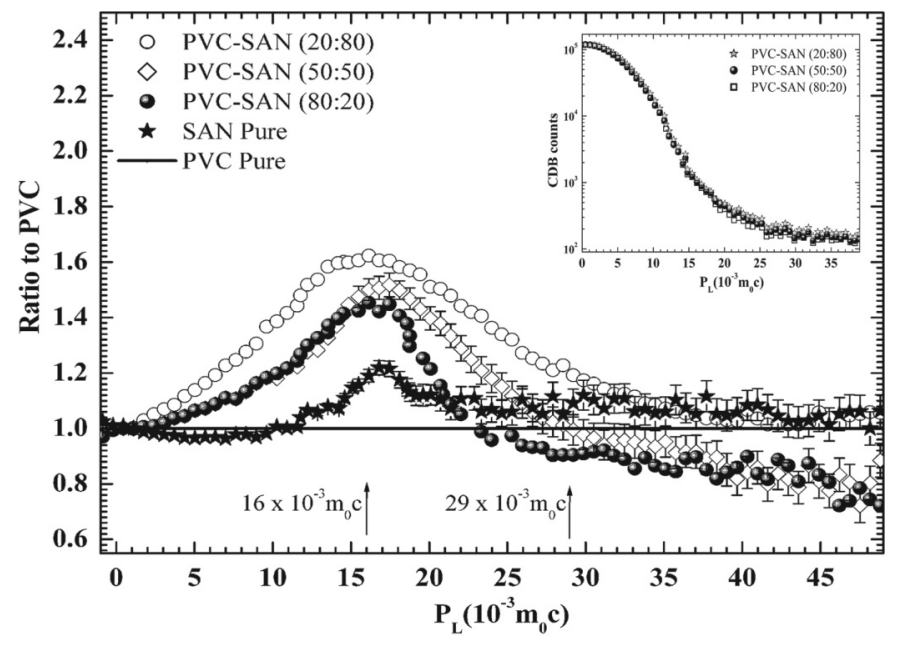

FIG. 3. Ratio curves for the CDB spectra of PVC-SAN blends and pure SAN with respect to pure PVC. In the inset CDB spectra of PVC-SAN blends with each spectrum normalized to the same total counts. 
considered as reference material. Figures 2 and 3 show the plot of momentum $\left(P_{L}\right)$ versus CDB ratio for PVC-EVA and PVC-SAN blends, respectively.

The contributions from different electron and positron states are evident in Fig. 2. Three regions are identified; the low momentum region from 0 to 3 momentum units corresponds to p-Ps annihilation and is generally observed as the narrow component of the CDB spectra. The intermediate momentum region from 3 to 13 momentum units corresponds to annihilation of $\sigma$ - and $\pi$-bond valence electrons of the polymer blend [30] and the high-momentum region above 15 momentum units corresponds to annihilation of core electrons with the positron of o-Ps [2].

The ratio curves exhibit non-Gaussian distributions for different compositions with different peak heights and widths. In contrast, the ratio curves in pure metals, with simple electronic structure, are readily approximated by Gaussian distributions. The peak heights infer that the probability of particular type of electrons participating in the annihilation process is high or low. Widening of the peak width can be attributed to contributions from p-Ps and o-Ps to annihilation [6].

The data from these graphs indicate the role of halogens and polar groups in the inhibition of the Ps formation process. In Fig. 2 the curves are narrow and peak around 17 units of momentum and show a rapid increases in the range from 7 to 17 units and decrease in the region from 18 to 29 units after which the curves for different compositions merge. The peak at 17 momentum units is in support of $e^{+}$trapping by the polar acetate group in EVA followed by annihilation with the core electrons of the unconjugated oxygen [2]. Similar observations were made by Nagai et al. for neutron-irradiated vitreous silica $\left(\mathrm{SiO}_{2}\right)$ [2] and for EMMA and PMMA polymers by Shantarovich et al. [1].

Furthermore, we observe from the momentum curves of PVC-EVA blend that the height of the distribution curve is proportional to the EVA concentration in the sample and the curve for 80 wt. \% of EVA in the blend results in a large peak height. On the other hand, as the PVC content increases (chlorine content in the blend increases), the curves become wider and the height decreases. This can be explained as follows: For the 20:80 composition of PVC-EVA, the peak is comparatively narrower and the height is large. Generally the narrow distribution is attributed to p-Ps since it is selfannihilation with low momentum electrons. On the other hand, the wider width is attributed to o-Ps annihilation because it annihilates via the pick-off process with surrounding electrons having higher momenta [6].

With this prescription, the $\mathrm{CDB}$ ratio peak at 17 units suggests positron trapping by the acetate group and hence annihilation with core electrons of oxygen. The widening of the ratio curves, as the PVC content increases or the EVA content decreases, is a sign of increased Ps inhibition, i.e., a decrease of the o-Ps intensity as revealed by the PLS data from Table I. Under these circumstances, the o-Ps in the system also annihilates noticeably with high momentum electrons since the ratio curves in the high momentum region are well above reference material curve values indicated by the solid line in Fig. 2. This result suggests further that compared to positron decay, positronium (Ps) is not preferentially captured by the polar acetate group $(-\mathrm{C}=\mathrm{O})$ because Ps is electrically neutral. Therefore, in the present blend, the contribution of the $-\mathrm{C}=\mathrm{O}$ group of EVA to Ps inhibition is not as strong as observed for the conjugated group [1]. Second, as the PVC content in the blend increases, the intensity of the peak height decreases indicating that positron trapping also decreases and the chlorine in the PVC inhibits the Ps formation [20,22], as evidenced by the observed decreased o-Ps intensity $\left(I_{3}\right)$, as shown in Table I, in complete agreement with earlier works [5,6,8,31-33].

For the second blend system PVC-SAN, we observe from Fig. 3 that the heights of the ratio curves are not to the scale observed for the first blend. As the SAN content increases, the height of the momentum distribution curve increases. The increase in the ratio of curve heights suggests an increase in positron, and even more Ps, annihilation due to the nearly nonpolar acrylonitrile (AN) group in SAN; however the probability of positrons being trapped in AN group is small as it is an extremely weak polar group. On the other hand, the SAN chains repel each other due to the AN group and the PVC chains can slide in between the SAN chains resulting in close packing of the chains. As can be seen, the o-Ps lifetime shown in Table I for the blend PVC-SAN with 20:80 composition exhibits longer lifetime (1.92 ns) compared to 80:20 composition in which PVC is $80 \%$ and o-Ps lifetime is shorter (1.78 ns). The PVC rich PVC-SAN blend (80:20) is known to be miscible [26] while the SAN rich blend (20:80) is known to be immiscible [26]. However, at least for now, electron momentum distribution cannot be directly connected to miscibility since we do not have enough data to make such an inference.

The wider distribution in the momentum region above 25 units could be interpreted as due to the charge neutrality of o-Ps and the electronegative nature of nitrogen in the $\mathrm{C} \equiv \mathrm{N}$ group. The ratio curves, beyond momentum range 25 units, approach reference lines or go well below it. This result suggests that the core electrons of nitrogen are not dominantly participating in o-Ps annihilation. With the increase in PVC content the curves get a little narrower and the peak height decreases. The explanation given for PVC-EVA blend holds good here too. The higher peak height in the PVC-SAN-20:80 composition, compared to other two compositions of the same blend, is indicative of slightly higher contributions of positrons, p-Ps, and o-Ps annihilation. As the PVC content increases, the inhibition of Ps formation also increases and hence results in reduced peak height as well as the width. The width of the momentum distribution curves spread from 16 to 29 units of momentum which is a spread of 13 units. The spread is on either side of the peak and could be seen as a sign of p-Ps with low and o-Ps annihilation through pick-off annihilation with marginally higher momentum electrons, respectively. In this case, positron trapping with the core electrons of $\mathrm{N}$ dominates. With increase in the PVC content, i.e., an increase in chlorine content, the momentum curves become narrower $[6,8]$ possibly due to an increase in the inhibition of Ps formation.

By comparison, the ratio of peak heights in Figs. 2 and 3 are different. For PVC-EVA the ratio is higher at 2.2 and lower in PVC-SAN at 1.6. The peaks around 17 units of momentum suggests oxygen in $\mathrm{C}=\mathrm{O}$ and nitrogen in $\mathrm{C} \equiv \mathrm{N}$ are contributing core/valence electrons of nearly almost equal 


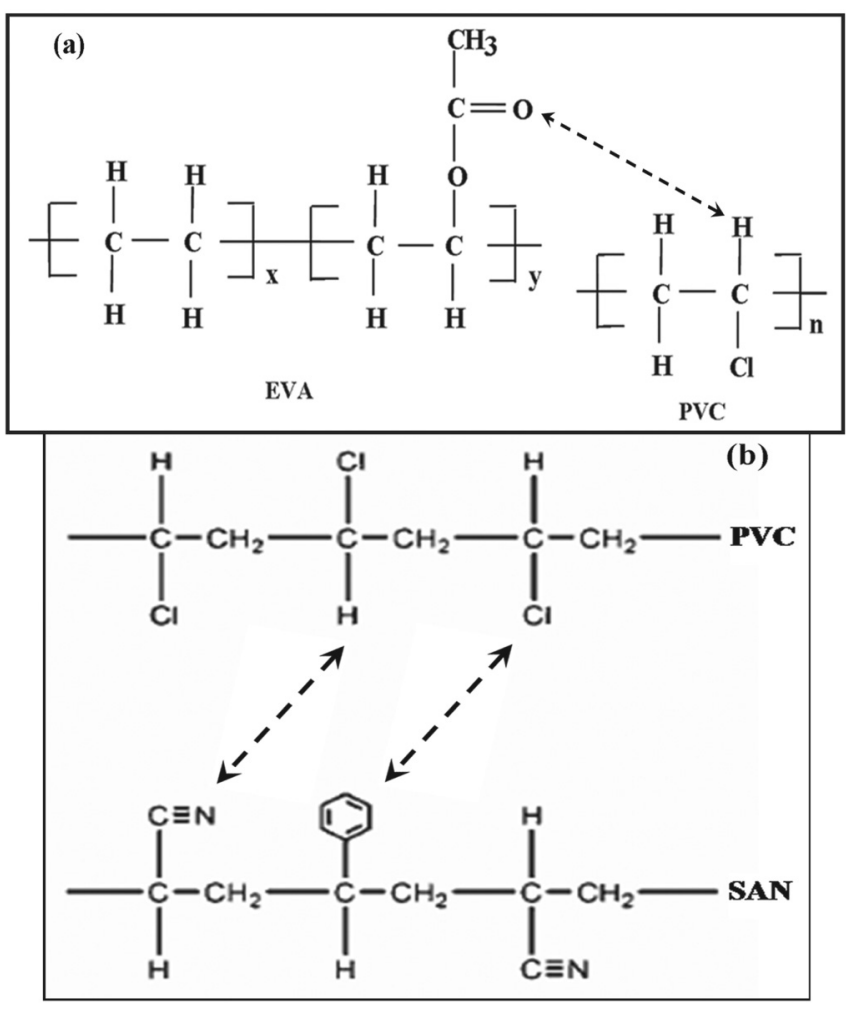

FIG. 4. Illustration of possible interaction in (a) PVC-EVA and (b) PVC-SAN polymer blends.

energy but oxygen core electrons are more effective in trapping of positrons in PVC-EVA than the valence electrons of nitrogen in PVC-SAN. As discussed in paper [1] the conjugated oxygen $(\mathrm{C}=\mathrm{C}-\mathrm{C}=\mathrm{O})$ is a strong inhibitor of Ps formation by trapping more positrons and not the unconjuagted oxygen $(-\mathrm{C}=\mathrm{O})$ as in the present samples. We infer that unconjugated oxygen is also an inhibitor of Ps formation but to a lesser degree which is clear in the positron lifetimes, discussed below. Since both blends contain PVC in which $\mathrm{Cl}$ is a known strong inhibitor of Ps formation [20,22], we observe the difference for the polar and nonpolar groups in the blends as the PVC content in the blend increases.

Similar to the pure EVA results, the ratio data for the SAN comes close to the pure PVC line and peaks around 16 units of momentum and hence similar arguments apply. Due to hydrgen bond formation between the $\mathrm{C} \equiv \mathrm{N}$ group of SAN and $\alpha$ hydrogen of PVC [see Fig. 4(b)] when SAN is blended with PVC, positron trapping increases at the electronegative $\mathrm{N}(3.04)$ site. In that way the electron of $\alpha$ hydrogen is pulled away from the hydrogen making it slightly more positive and the electron density around $\mathrm{N}$ increases. Therefore the positron trapping increases and we observe the ratio curve to peak around 16 units. Precisely similar to the previous blend, as the SAN content in the blend increases, we increase the number of interaction sites and positron trapping is enhanced, and the peak height increases and vice versa. The PVC-SAN blend is shown to be partially miscible [26] due to the mechanical interactions, i.e., the repulsion between the chains due to acrylonitrile groups of SAN, as explained further in the next section.

\section{B. PALS results}

The results of the three component analyses of positron lifetime spectra in pure polymers and their two blend systems are summarized in Table I. The attribution of the three lifetime components and their intensities was given earlier. Only the o-Ps lifetime and its intensity are considered since the focus of this paper is on the inhibition of Ps formation in the blends. As can be seen from Table I, the o-Ps intensity $I_{3}$ value in pure PVC is only $6.2 \%$ compared to the other two component polymers in which it is around $20 \%$. This is a clear indication that the presence of halogen in the polymer strongly inhibits the Ps formation [20,22]. To understand the chemical environment from which positron and Ps annihilation occurs in these two polymer blends, let us look at the possible interactions between the components. Though the PVC-EVA system is a known immiscible blend [24,26], there is a slight possibility of interaction between the components through the carbonyl group $(\mathrm{C}=\mathrm{O})$ of acetate in EVA and the $\alpha$-hydrogen of PVC $[34,35]$ as depicted in Fig. 3(a). The $\mathrm{C}$ and $\mathrm{O}$ form $s p^{2}-s p^{2} \sigma$ bonds. The two remaining $s p^{2}$ orbitals of carbon are attached to alkyl or aryl groups or hydrogen. The other two $s p^{2}$ orbitals of oxygen remain as lone (unshared) pairs. Both $\mathrm{C}$ and $\mathrm{O}$ have an unhybridized $2 p$ orbital containing one electron.

Lateral overlap of these two orbitals produces a $\pi$ molecular orbit containing two electrons. In the $\mathrm{C}=\mathrm{O}$ bond, the electron sharing is unequal because oxygen is more electronegative than the carbon leading to polarization of the $\pi$-electron cloud towards oxygen so it is a strong polar molecule. On the other hand, PVC-SAN is a partially miscible blend [26]. In this blend intramolecular repulsion between segments of SAN (mainly due to the AN group) accounts for the partial miscibility of this blend [36]. The interaction between SAN and PVC is influenced by the acrylonitrile (AN) concentration in SAN due to self-association of very weak polar AN groups and steric effects. Also there are two more possible interactions, namely an induced dipole interaction between the aromatic quadrupole (benzene ring) of SAN and halogen (chlorine) of the PVC as well as hydrogen bonding between the AN and the $\alpha$-hydrogen of PVC [37] as shown in Fig. 4(b). To understand the type of electrons participating in annihilation and hence the momentum distribution ratio curves observed, let us consider the weak polar acrylonitrile group with the chemical formula $\mathrm{C}_{3} \mathrm{H}_{3} \mathrm{~N}$ which has 20 valence electrons resulting in $6 \sigma$ and $3 \pi$ bonds. It is an example of connecting molecular fragments together where delocalization of $\pi$ electron density can occur over more than two atoms. The distribution of mass and the architecture of acrylonitrile can be described by the joining of an ethenyl radical $\left(\mathrm{CH}_{2}=\mathrm{CH}\right)$ with the cyanidyl radical $(\mathrm{C} \equiv \mathrm{N})$ using a single two electron covalent bond. In condensed formulation, acrylonitrile can be written as $\mathrm{CH}_{2}=\mathrm{CH}-\mathrm{C} \equiv \mathrm{N}$ : where there is a $\sigma \mathrm{MO}$, containing two electrons, that bonds the fragment radicals (ethenyl and cyanidyl) together. The electron energy correlation diagram traces the overlap interactions of the fragment $\sigma$ and $\pi \mathrm{MO}$ that lead to resonance for the relevant MOs of acrylonitrile. This provides a partial explanation for the concept of $\pi$ conjugation or delocalization. The two filled orbitals having the same energy as in the cyanidyl fragment corresponds, respectively, to a nonbonding and a $\pi$-bonding 
MO carried over into the acrylonitrile MO description. The MO labeled $\pi$ MO is the most interesting one because it is the bonding orbital that has a different energy correlation as a result of conjugation.

For acrylonitrile, the delocalization of the two electrons in the $\pi$ MO results when the $\pi$-bonding MO of the ethenyl radical interact with a parallel $\pi$-bonding MO of the cyanidyl radical to create a new super $\mathrm{MO}$ which allow electron density to be spread over more than two atoms. In this case the filled $\pi$-bonding MO having an unchanged energy becomes a nonbondinglike MO that has a perpendicular orientation with respect to the bonding super MO of acrylonitrile. Each bonding MO is occupied by two electrons but the $\pi$ conjugated electrons are spread out over more than two atoms in a linear super MO while the remaining filled MOs are localized on two atoms, namely $\mathrm{C}$ and $\mathrm{N}$. As the nitrogen is more electronegative (3.04) than the carbon (2.55), the annihilation will be more with core electrons of nitrogen than with $\mathrm{C}$ which is apparent from the peak at 16 units of momentum in momentum distribution curves for this blend.

However, from the point of view of inhibition of Ps formation, the o-Ps intensity $\left(I_{3}\right)$ is the parameter we need to consider since it represents the probability of Ps formation in a system. As we can see from Table $\mathrm{I}, I_{3}$ decreses by $\sim 12.2 \%$ for the PVC-EVA blend while it decreases by $9.2 \%$

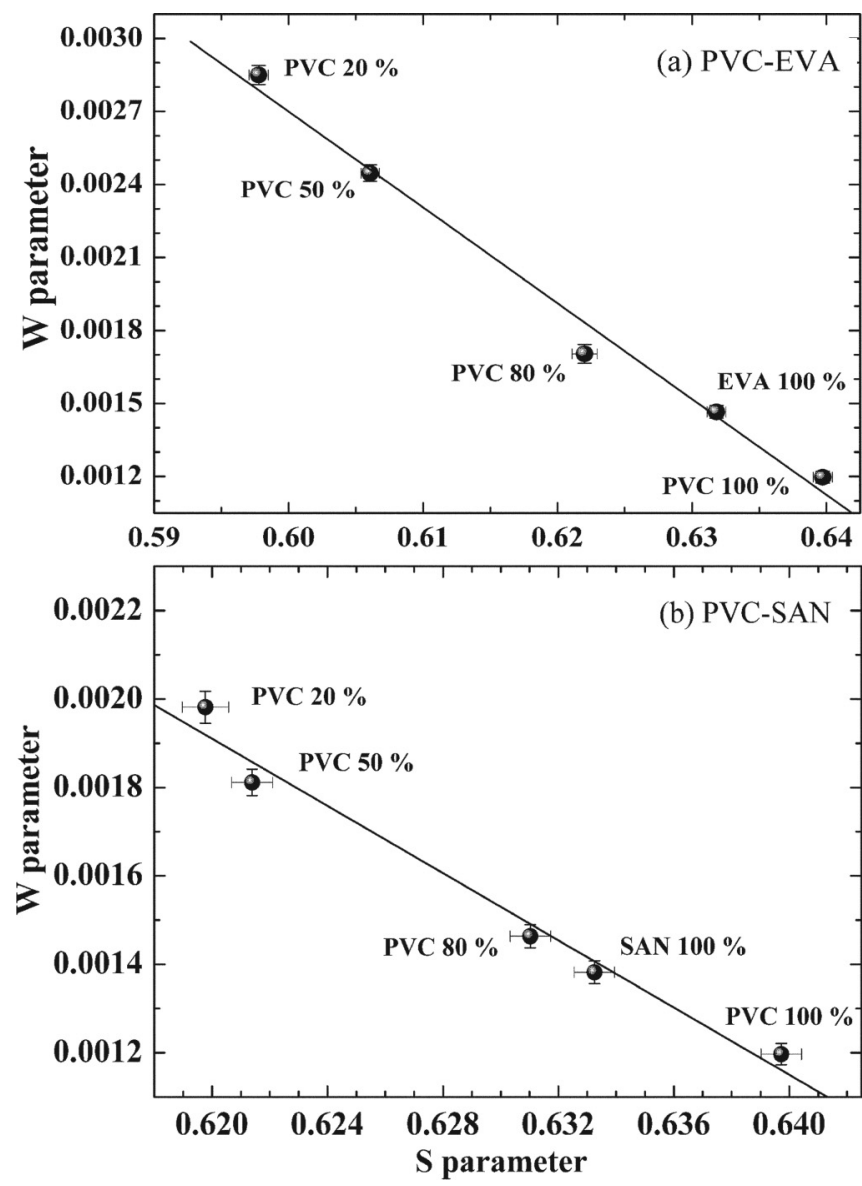

FIG. 5. Correlation between the $S$ and $W$ parameters at different compositions of (a) PVC-EVA and (b) PVC-SAN polymer blends. for the PVC-SAN blend when the chlorine percentage in the blend increases. This result is in excellent agreement with the literature data for systems containing chlorine [20]. From these values it becomes clear that there is difference of $3 \%$ in the decrease in o-Ps intensity between blends containing EVA and those containing SAN. Therefore, the inhibiting ability of unconjugated oxygen from the EVA acetate group is really noticeable from 50:50 blends of PVC-EVA and PVC-SAN. In the two blends with this composition, PVC is of the same amount but we observe decrease in $I_{3}$ is about $5.4 \%$ more in PVC-EVA (19\% to $8.9 \%$ ) than in PVC-SAN $(18.7 \%$ to $14.3 \%$ ). Therefore, the role of the polar group, namely the unconjugated oxygen in EVA, is very clear from this. In PVC-EVA the inhibiting action is from the chlorine of PVC and the unconjugated oxygen of EVA. In both blends, there is a greater probability for the formation of a $\mathrm{Cl}^{-}-e^{+}$ bound state which prevents the possibility of positrons being attracted to an electron to form Ps. If the nitrogen of SAN was also effective as an inhibitor of Ps formation, then we could have observed that the net decrease in $I_{3}$ would have been similar to the oxygen on EVA. Since the $\mathrm{C} \equiv \mathrm{N}$ of SAN is nonpolar (or very weakly polar) it is incapable of inhibiting Ps formation thus clearly showing the role of the polar groups.

\section{Line shape parameters $S$ and $W$}

The above arguments are supported further by the line shape parameters $S$ and $W$ obtained from CDB data. These are

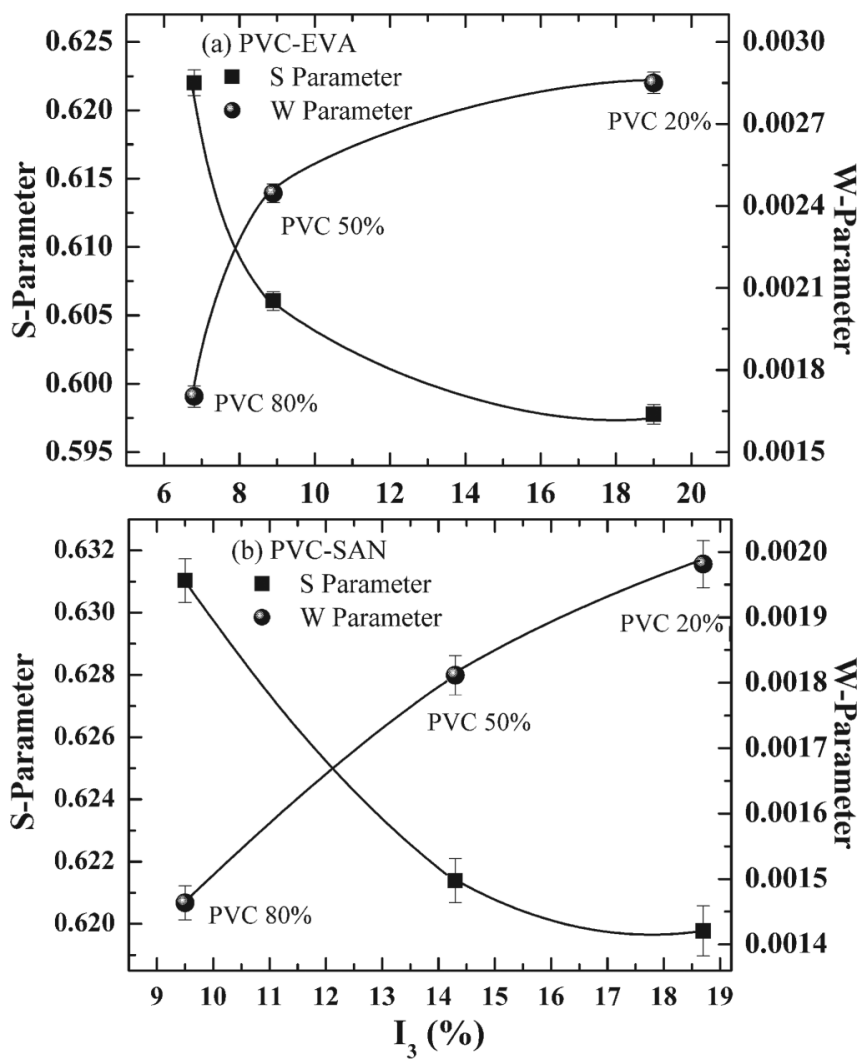

FIG. 6. Plot of $S$ and $W$ parameters as a function of PVC wt. \% in (a) PVC-EVA and (b) PVC-SAN polymer blends at varying compositions. 
shown in Figs. 5(a) and 5(b), respectively, for PVC-EVA and PVC-SAN. Using the standard interpretation of the conventional $S$ and $W$ parameters [2,9,38], the plots producing straight line graphs suggest the presence of only one type of vacancy or open space in the region where the positrons annihilate. For polymers and blends the free volume cavities are larger vacancies than the crystalline defects. Free volume cavities are a measure of the fraction of o-Ps annihilation with core electrons, possibly with oxygen core electrons of the EVA [2]. If more positrons or Ps annihilate with the oxygen, the CDB spectrum will result in a larger $W$ parameter [2]. It is interesting to observe for both blend systems in which PVC is a constituent that, as the PVC content increases, the $\mathrm{W}$ parameter decreases indicating less o-Ps annihilation and hence more inhibition of Ps formation.

In Fig. 6(a) we plot $S$ and $W$ parameters as a function of o-Ps intensity $\left(I_{3}\right)$. It can be seen that the $W$ parameter increases as $I_{3}$ increases, which clearly points to the type of electrons which annihilate with o-Ps. The $W$ parameter is defined to characterize high momentum electrons, some of which could be core electrons of oxygen. Also the higher value of $I_{3}$ implies the chlorine content is less and inhibition is also less. The decrease of the $S$ parameter implies that positron trapping by oxygen also decreases. From Fig. 6(b) we observe a similar trend as in the PVC-EVA blend but the magnitude of $W$ is reduced. The increase in the SAN content means an increase in $I_{3}$ shows that $W$ increases while $S$ decreases. Here the higher values for the $W$ parameter indicate that more o-Ps annihilates in the SAN domain, probably with the core (valence) electrons of nitrogen.

\section{CONCLUSION}

The positron annihilation results for the two blends were derived from measurements of the coincidence Doppler broadening spectroscopy and positron annihilation lifetime spectroscopy. A higher probability for annihilation of positrons (by trapping) and positronium with core electrons of the strong polar acetate groups in EVA (unconjugated oxygen) results in narrow higher momentum peaks. The very weak polar group $\mathrm{C} \equiv \mathrm{N}$ in $\mathrm{SAN}$ (core/valence electrons of nitrogen since it is more electronegative than carbon) results in wider momentum distribution curves. The polar groups in binary polymer blends inhibit Ps formation but the effect is less in comparison with a strong inhibitor like a halogen. Further support for this result is derived from the results of the blend with a nonpolar (very weak polar) group namely $\mathrm{C} \equiv \mathrm{N}$ in $\mathrm{SAN}$. The varying peak heights of the momentum distribution curves are clearly due to enhanced positron trapping around the oxygen and nitrogen atoms due to hydrogen bond formation, respectively, in PVCEVA and PVC-SAN blends. We conclude that the coincidence Doppler broadening measurements provide more insight in understanding the hydrogen bond formation in binary blends than could be obtained from positron lifetime measurements.

\section{ACKNOWLEDGMENTS}

One of the authors (P. Ramya) thanks the University Grants Commission, New Delhi, for providing support through a RFSMS fellowship during this work. Funding was obtained from the Australian Research Council and The University of Western Australia.
[1] V. P. Shantarovich, T. Suzuki, C. He, and V. W. Gustov, Rad. Phys. Chem. 67, 15 (2003).

[2] Y. Nagai, T. Nonaka, M. Hasegawa, Y. Kobayashi, C. L. Wang, W. Zheng, and C. Zhang, Phys. Rev. B 60, 11863 (1999).

[3] Y. Kobayashi, C. L. Wang, K. Hirata, W. Zheng, and C. Zhang, Phys. Rev. B 58, 5384 (1998).

[4] H. Chunqing, V. P. Shantarovich, T. Suzuki, S. V. Stepanov, R. Suzuki, and M. Matsuo, J. Chem. Phys. 122, 214907 (2005).

[5] A. Baranowski, M. Debowska, K. Jerie, R. Girulska, Z. Pawelka, and R. T. Sicorski, J. Rad. Nucl. Chem. 190, 463 (1995).

[6] K. Sato, K. Ito, K. Hirata, R. S. Yu, and Y. Kobayashi, Phys. Rev. B 71, 012201 (2005).

[7] K. Sato, D. Shanai, Y. Hotani, T. Ougizawa, K. Ito, K. Hirata, and Y. Kobayashi, Phys. Rev. Lett. 96, 228302 (2006).

[8] K. Sato, K. Ito, K. Hirata, R. S. Yu, and Y. Kobayashi, Rad. Phys. Chem. 76, 112 (2007).

[9] N. Djourelov, C. He, T. Suzuki, V. P. Shantarovich, Y. Ito, K. Kondo, and Y. Ito, Rad. Phys. Chem. 68, 689 (2003).

[10] U. Myler and P. J. Simpson, Phys. Rev. B 56, 14303 (1997).

[11] T. Suzuki, C. He,V. P. Shantarovich, K. Kondo, E. Hamada, M. Matsuo, L. Ma, and Y. Ito, Rad. Phys. Chem. 66, 161 (2003).

[12] N. Djourelov, T. Suzuki, Y. Ito, V. Shantarovich, Y. Ito, K. Kondo, and J. Onoe, Chem. Phys. 298, 183 (2004).

[13] N. Djourelov, T. Suzuki, R. S. Yu, V. Shantarovich, and K. Kondo, Chem. Phys. 302, 179 (2004).
[14] G. Dlubek, K. Saarinen, and H. M. Fretwell, Nucl. Instrum. Methods Phys. Res., Sect. B 142, 139 (1998).

[15] K. Ito, Y. Kobayashi, and A. Nanasawa, Appl. Phys. Lett. 82, 654 (2003).

[16] Y. Ito, K. I. Okamoto, and K. Tanaka, J. Phys. IV 3, 241 (1993).

[17] K. G. Lynn, J. R. Mac Donald, R. A. Boie, L. C. Feldman, J. D. Gabbe, M. F. Robbins, E. Bonderup, and J. Golovchenko, Phys. Rev. Lett. 38, 241 (1977).

[18] Y. C. Jean, Microchem. J. 42, 72 (1990).

[19] J. Liu, Y. C. Jean, and H. Yang, Macromolecules 28, 5774 (1995).

[20] J. A. Merrigan, J. H. Green, S. J. Tao, in Techniques of Chemistry1, Part III D, Physical Methods of Chemistry, edited by A. Weissberger and B. W. Rossiter (Wiley Interscience, New York, 1972).

[21] C. Ranganathaiah and G. N. Kumarswamy, J. Appl. Polym. Sci. 111, 577 (2009).

[22] D. Meghala and C. Ranganathaiah, Polymer 53, 842 (2012).

[23] H. B. Ravikumar, C. Ranganathaiah, G. N. Kumarswamy, and S. Thomas, Polymer 46, 2372 (2005).

[24] J. M. Raj and C. Ranganathaiah, J. Polym. Sci., Part B: Polym. Phys. 47, 619 (2009).

[25] A. M. A. M. Altaweel, C. Ranganathaiah, and B. Kothandaraman, J. Adhes. 85, 200 (2009).

[26] P. Ramya, C. Ranganathaiah, and J. F. Williams, Polymer 53, 4539 (2012). 
[27] P. Asoka-Kumar, M. Alatalo, V. J. Ghosh, A. C. Kruseman, B. Nielsen, and K. G. Lynn, Phys. Rev. Lett. 77, 2097 (1996).

[28] M. H. Weber, Mater. Sci. Forum 629, 363 (2001).

[29] P. Kirkegaard, N. J. Pederson, and M. Eldrup, Riso Nat Lab Reports, M-2740, 5 (1989).

[30] S. Berko, R. E. Kelley, and J. S. Plaskett, Phys. Rev. A 106, 824 (1957).

[31] G. Wikander, Chem. Phys. Lett. 80, 361 (1981).

[32] O. E. Mogensen, J. Chem. Phys. 60, 998 (1974).

[33] S. J. Tao, Appl. Phys. 10, 67 (1976).
[34] D. Feldman and M. Rusu, Eur. Polym. J. 10, 41 (1974).

[35] E. Corradini, A. F. Rubira, and E. C. Muniz, Eur. Polym. J. 33, 1651 (1997).

[36] C. H. Kim, J. K. Park, and T. S. Hwang, Polym. Eng. Sci. 36, 535 (1996).

[37] H. S. Moon, W. M. Choi, M. H. Kim, and O. O. Park, J. Appl. Polym. Sci. 104, 95 (2007).

[38] R. Zhang, J. Robles, and J. Kang, Macromolecules 45, 2434 (2012). 